\title{
Long-acting liposomal bupivacaine and postoperative opioid use after Peyronie's disease surgery: a pilot study
}

\author{
Cameron J. Britton, Bridget L. Findlay, Niki Parikh, Tobias Kohler, Sevann Helo, Matthew J. Ziegelmann \\ Department of Urology, Mayo Clinic, Rochester, MN, USA \\ Contributions: (I) Conception and design: MJ Ziegelmann; (II) Administrative support: MJ Ziegelmann; (III) Provision of study materials or patients: \\ MJ Ziegelmann, T Kohler, S Helo; (IV) Collection and assembly of data: CJ Britton, MJ Ziegelmann; (V) Data analysis and interpretation: CJ \\ Britton, MJ Ziegelmann; (VI) Manuscript writing: All authors; (VII) Final approval of manuscript: All authors. \\ Correspondence to: Matthew J. Ziegelmann, MD. Mayo Clinic, 200 First Street SW, Rochester, MN 55905, USA. Email: Ziegelmann.Matthew@mayo.edu.
}

\begin{abstract}
Background: Novel strategies have been proposed to minimize postoperative opioid use, yet many patients experience significant pain after penile surgery. Our objective was to evaluate postoperative opioid use in patients undergoing penile ring block with long-acting liposomal bupivacaine (LB; Exparel) during surgery for Peyronie's disease (PD).

Methods: We identified patients who underwent tunica albuginea plication (TAP) and plaque excision/ grafting (PEG) for PD between July 2019 and September 2020. Intraoperatively, a ring block was administered at the penile base penis with $20 \mathrm{cc}$ of LB. Patients were instructed to use over the counter pain medications as first line treatment for postoperative pain, and opioids were available for severe breakthrough pain as needed [ 7.5 oral morphine equivalents (OME) $=5 \mathrm{mg}$ oxycodone]. Opioid use was assessed during the first five days postoperatively.

Results: In total, 28 patients met inclusion criteria including 18/28 (64\%) who underwent TAP and 10/28 (36\%) who underwent PEG. Median patient age was 56 years (IGR 51;61). Median postoperative 10-point visual analogue pain score was 0 (range $0-3$ ). Duration of penile anesthesia ranged from 1.5-4 days. In total, 9/28 patients (32\%) utilized opioids during the first five days postoperatively (range 7.5-75 OME). Two patients (7\%) required opioids during the first two days after surgery. 27/28 (96\%) were satisfied or highly satisfied with postoperative pain control.

Conclusions: Intraoperative penile ring block with LB resulted in excellent pain control with local anesthetic duration of 1.5-4 days. The majority of patients did not require any opioids during the early postoperative period. Further study comparing outcomes with shorter-acting local anesthetics is necessary to balance pain control benefits with additional cost.
\end{abstract}

Keywords: Plication; incision and grafting; Xiaflex; collagenase; penile traction

Submitted Apr 22, 2020. Accepted for publication Oct 26, 2020.

doi: 10.21037/tau-20-871

View this article at: http://dx.doi.org/10.21037/tau-20-871

\section{Introduction}

Standardized pain management strategies following penile surgery are lacking and opioids are routinely prescribed postoperatively. This has unintended and far-reaching consequences. The opioid overdose death rate in the United States has significantly increased over the past three decades, and up to $1 / 3^{\text {rd }}$ of these deaths are linked to prescription opioid medications (1). Several studies have specifically identified a positive correlation between postoperative opioid prescriptions, overdose, and new-onset dependence in patients without prior opioid exposure or substance abuse history (2-4). Among patients undergoing urologic procedures, the risk for prolonged opioid use is estimated at 2-9\% (5-9). 
"Enhanced recovery after surgery" (ERAS) pathways focus on non-opioid interventions. They have been proposed to shorten hospital stays and combat the opioid epidemic (10-12). Local anesthesia with agents including lidocaine, prilocaine, bupivacaine, and ropivacaine is a standard component of many ERAS protocols. These anesthetics have estimated half-lives of $1.5-8$ hours, thereby limiting prolonged analgesia beyond 12-24 hours for most patients $(13,14)$. Liposomal bupivacaine (LB; Exparel ${ }^{\circledR}$; Pacira Biosciences Inc. Parsippany, NJ) was developed as alternative, longer-acting local anesthetic with effects lasting up to 96 hours (15). Two pivotal randomized controlled trials comparing liposomal bupivacaine to placebo identified improvements in postoperative pain scores for 72 hours after surgery and significant reductions in opioid consumption with LB $(16,17)$. Within the realm of urologic surgery, there is a paucity of data on the benefits of long-acting local anesthetics including LB. This is highly relevant to penile and scrotal procedures where early prominent pain rarely persists beyond the first several days postoperatively (18).

Penile plication and plaque incision (or partial excision) and grafting are well established surgical approaches to treat penile curvature in men with Peyronie's Disease (PD) (19-22). Both procedures are successful in achieving penile straightening and result in satisfactory outcomes for the majority of appropriately selected patients. As with any intervention, postoperative pain control remains a common and significant concern after PD surgery. Techniques for traditional dorsal penile nerve block or penile ring block with short-acting local anesthetics during penile surgical procedures have been previously described $(23,24)$. However, to our knowledge, no report has specifically assessed postoperative outcomes after penile ring block using LB at the time of penile surgery for PD. Here, we sought to evaluate our experience with postoperative pain control including the use of opioid medications in patients who received LB during penile straightening surgery for PD. We hypothesized that LB would minimize the need for oral opioid pain medications in the majority of patients. We present the following article in accordance with the STROBE reporting checklist (available at http://dx.doi. org/10.21037/tau-20-871).

\section{Methods}

The study was conducted in accordance with the Declaration of Helsinki (as revised in 2013). The study was approved by institutional review board of Mayo Clinic (No. 20-003836) and informed consent was taken from all individual participants. We reviewed our prospectively maintained clinical database for all patients undergoing surgical intervention for PD by a single surgeon (MJZ) between July 2019 and September 2020. Retrospective chart review was performed to identify pertinent patient demographics including preoperative clinical assessment, intraoperative findings, and postoperative outcomes. The study cohort included those adult male patients $(>18$ years of age) who underwent intraoperative penile ring block with bupivacaine liposome injectable suspension (Exparel; Pacira Biosciences, Inc; Parsippany, NJ, USA) for local anesthesia during tunica albuginea plication (TAP) or partial plaque excision and grafting (PEG). Patients who underwent concurrent penile prosthesis placement or had a personal history of substance abuse (including recent opioid exposure within the preceding 6 months) were excluded.

\section{Preoperative considerations}

Prior to treatment, all patients underwent a thorough history and physical examination to establish the diagnosis of $\mathrm{PD}$ as recommended the American Urological Association PD guideline panel (22). In those patients who desire definitive treatment, a formal curvature assessment and penile duplex Doppler ultrasonography (PDDU) is performed. Patients with PD are extensively counseled on all treatment options including oral medications, penile traction, intralesional injections, and surgical straightening with or without a penile prosthesis (22). A surgical algorithm is employed similar to that originally described by Levine and Lenting (21).

Tunica Albuginea Plication (TAP), via a modified Baskin-Duckett-Levine approach, is used for patients with adequate baseline penile length, curvature $<60-70$ degrees, and absence of severe indentation or hourglass deformity with hinge-effect (defined as penile instability with the application of axial loading pressures) (19,25). Partial plaque excision and grafting (PEG) using a Tutoplast-processed human pericardial allograft (Coloplast Corp., Minneapolis, $\mathrm{MN}$ ) is offered for patients with more severe curvature and prominent baseline indentation/hourglass (20). All patients who elect surgical straightening are extensively counseled regarding postoperative outcomes including potential for temporary or permanent sensory loss, erectile dysfunction, and suture palpability (plication). 


\section{Intraoperative penile ring block (Figure 1)}

Preoperative pain scores (visual analog pain scores on a scale from 0-10) were obtained in the holding area prior to the procedure. In patients undergoing TAP for dorsal, lateral, or dorsolateral curvature, a ventral midline incision is made along the penile shaft median raphe in the area of maximum curvature. In contrast, for patients with ventral penile curvature undergoing plication, and for all patients undergoing partial plaque excision and grafting, a circumcising incision is used. To avoid compromise to the surgical planes, local anesthesia is not administered until the cessation of the case, just prior to surgical dressing placement. At this point, a circumferential penile ring block is carried out at the base of the penis with the penis on gentle stretch. A total of $20 \mathrm{cc}$ of liposomal bupivacaine is injected. First, approximately $8 \mathrm{cc}$ is administered dorsally, taking care to skive along the neurovascular bundle (Figure 1A). An additional $4 \mathrm{cc}$ is administered laterally on each side with the needle directed adjacent to the corpora to ensure adequate administration to the lateral (circumflex) dorsal penile nerve branches (Figure $1 B$ ). Finally, $4 \mathrm{cc}$ is injected ventrally just superficial to the urethra which is identified by palpation (Figure 1C).

\section{Postoperative follow-up}

All patients are assessed in the postoperative recovery room at which time pain scores are reassessed (26). Patients are dismissed to home the same day with instructions to employ a pain regimen of acetaminophen (625-1,000 mg every 6 hours), ibuprofen (200-400 mg every four to six hours), and twice daily application of ice to the groin for the first 5 days after surgery unless contraindicated. A prescription for a small quantity of oxycodone $5 \mathrm{mg}$ tablets (10 tabs total) is also provided, and patients are instructed to use this only for breakthrough pain not controlled by over the counter analgesics.

As part of our standard postoperative follow-up, patients are contacted via telephone or electronic medical recordbased correspondence between postoperative days \#5-7, and all patients are seen in clinic (or via telemedicine) for initial follow-up at or around postoperative day \#14. Postoperative follow-up included assessment of postoperative opioid utilization (number of tablets) and subjective perception of postoperative pain control.

\section{Analysis}

The primary outcome of interest was postoperative opioid use (number of tablets). Secondary outcomes included patient-perceived duration of penile anesthesia after the penile ring block, subjective perception of pain control, timing of opioid use (if applicable), and opioid refills. Secondary clinical characteristics and patient demographics were described using medians [interquartile ranges (IQR)] and percentages for continuous and discrete variables, respectively. Chi-square/Fisher's exact tests and Wilcoxon signed-ranked tests were used to compare treatment-related outcomes as indicated. Unless otherwise noted, p-values were two-sided, with $\mathrm{P}<0.05$ considered statistically significant. No power analysis was performed due to the small cohort included in this early pilot study.

\section{Results}

A total of 28 patients met inclusion criteria including 18/28 (64\%) who underwent TAP and 10/28 (36\%) who underwent PEG. Median patient age was 56 (IQR 51;61). Table 1 displays an overview of baseline patient cohort demographics. Median immediate postoperative 10-point visual analogue pain score was 0 (range $0-3$ ). There were no adverse intraoperative events resulting from LB administration.

Median total postoperative oxycodone use within the first five days was 0 tablets (range $0-10$ tablets). In total, $9 / 28$ patients $(32 \%)$ used any opioids. Only two patients reported using any oxycodone within the first two days postoperatively. 32 oxycodone tablets were consumed out of a total of 280 tablets prescribed (11\%). No patient required an opioid refill, and only a single patient required opioids beyond postoperative day five in the context of painful nocturnal erections after TAP to treat 90-degree congenital penile curvature (patient \# 21 in Table 2). Median duration of penile anesthesia related to the block was 3 days (range 1.5-4). At two-week follow-up, 27/28 patients reported satisfaction with postoperative pain control (96\%).

Further details regarding patient-specific opioid use are seen in Table 2. Patients who used penile traction therapy preoperatively were significantly more likely to require postoperative opioids $(\mathrm{n}=5 / 6,83 \%)$ compared with those who did not use penile traction prior to surgery $(n=4 / 22$, $18 \% ; \mathrm{P}=0.029)$. In contrast, there was no significant 

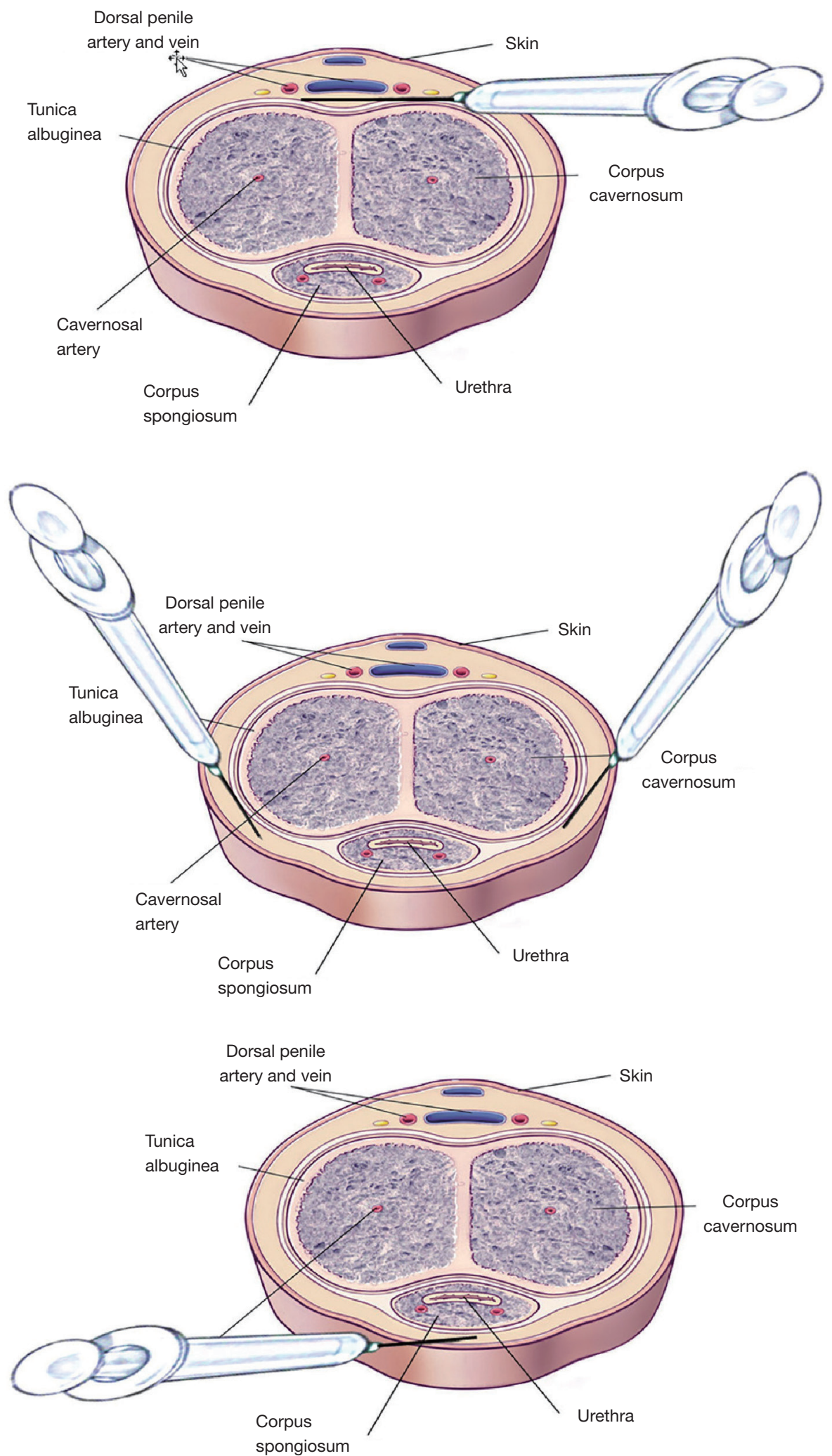

Figure 1 Illustration demonstrating our technique for penile ring block involved injection of local anesthesia along the dorsal (A), lateral (B), and ventral (C) aspects of the penile shaft. 
Table 1 Patient baseline demographics

\begin{tabular}{|c|c|}
\hline & $\begin{array}{l}\text { Total cohort } \\
\quad(n=28)\end{array}$ \\
\hline Age, median (range) & $56(18-70)$ \\
\hline PD symptom duration (months), median (range) & $24(9-72)$ \\
\hline Baseline subjective erectile dysfunction*, n (\%) & $6(21)$ \\
\hline Subjective penile shortening, $\mathrm{n}(\%)$ & $23(82)$ \\
\hline Preceding penile trauma, $\mathrm{n}(\%)$ & $8(29)$ \\
\hline \multicolumn{2}{|l|}{ Medical comorbidities, n (\%) } \\
\hline Diabetes & $4(14)$ \\
\hline Hypertension & $8(29)$ \\
\hline Hyperlipidemia & $6(21)$ \\
\hline Coronary artery disease & $1(4)$ \\
\hline \multicolumn{2}{|l|}{ Prior PD treatments } \\
\hline Intralesional collagenase, $\mathrm{n}(\%)$ & $10(36)$ \\
\hline Penile traction, n (\%) & $6(21)$ \\
\hline \multicolumn{2}{|l|}{ Objective curvature assessment } \\
\hline Baseline curvature (degrees), median (range) & $60(35-105)$ \\
\hline \multicolumn{2}{|l|}{ Curvature direction, n (\%) } \\
\hline Dorsal & $6(21)$ \\
\hline Ventral & $3(11)$ \\
\hline Lateral & $2(7 \%)$ \\
\hline Biplanar & $17(61)$ \\
\hline Indentation/hourglass deformity, n (\%) & $16(57)$ \\
\hline Hinge-effect, n (\%) & $11(39)$ \\
\hline \multicolumn{2}{|l|}{ Surgery performed, n (\%) } \\
\hline Tunica albuginea plication & $18(64)$ \\
\hline Partial plaque excision and grafting & $10(36)$ \\
\hline
\end{tabular}

*, Responsive to oral phosphodiesterase-5 inhibitors.

difference in postoperative opioid use based on age, prior intralesional therapy, medical comorbidities, history of penile trauma, and the type of procedure performed $(\mathrm{P}>0.05$ for all). At the most recent follow-up, two patients reported bothersome diminished erectile rigidity and penile shaft sensory loss after TAP $(n=1)$ and PEG $(n=1)$.

\section{Discussion}

Postoperative opioid consumption, even in patients without a history of substance abuse, may lead to persistent use in up to $8 \%$ of patients (5-9). This has important implications when considering the vast overprescribing seen after urologic surgeries $(27,28)$. Recent progress is attributed to heightened awareness, education efforts, and novel pain management strategies. Local anesthetics are an important aspect of the postoperative pain control regimen, but most agents have a relatively short duration of action. This may lead to satisfactory pain control in the post-anesthesia unit, but necessitates alternative means, including oral opioids, as the local effects diminish at home. Long-acting local anesthetics, on the contrary, provide a greater duration of action, mitigating the need for alternative agents. In the current study we found that nearly $70 \%$ of patients were able to avoid any postoperative opioid use after penile plication or grafting procedures for PD when a penile ring block with LB was performed. To our knowledge, this is the first study to specifically evaluate outcomes with this agent in men undergoing surgery for PD, and our findings suggest that LB is an excellent option for controlling perioperative pain with penile surgery.

The devastating effects of the opioid epidemic have prompted various interventions, including prescribing guidelines and enhanced recovery after surgery (ERAS) pathways (10-12). Recently, Lucas et al. evaluated the combination of acetaminophen, meloxicam or celecoxib, gabapentin, and penile/pudendal nerve blocks with local anesthetic (using a mixture of lidocaine and bupivacaine) compared to an opioid-based pain management regimen following inflatable penile prosthesis procedures (29). Using this protocol, the authors found a significant decrease in postoperative pain coupled with lower oral opioid consumption when compared with a historical opioidbased regimen. There are new concerns regarding potential adverse perioperative effects with gabapentinoids including pulmonary complications (30,31). At our institution, colleagues in anesthesia have expressed similar concerns. This has prompted us to re-evaluate and limit our use of gabapentinoids in the preoperative and perioperative period, particularly at higher doses. Local anesthetic nerve blocks are another cornerstone of ERAS pathways designed to provide immediate perioperative and postoperative pain relief (32-34). However, standard local anesthetics such as the lidocaine/bupivacaine mixture used by Lucas et al. have a relatively limited duration of action, thereby compromising their ability to provide prolonged pain relief beyond the first several hours postoperatively $(13,14)$. LB 


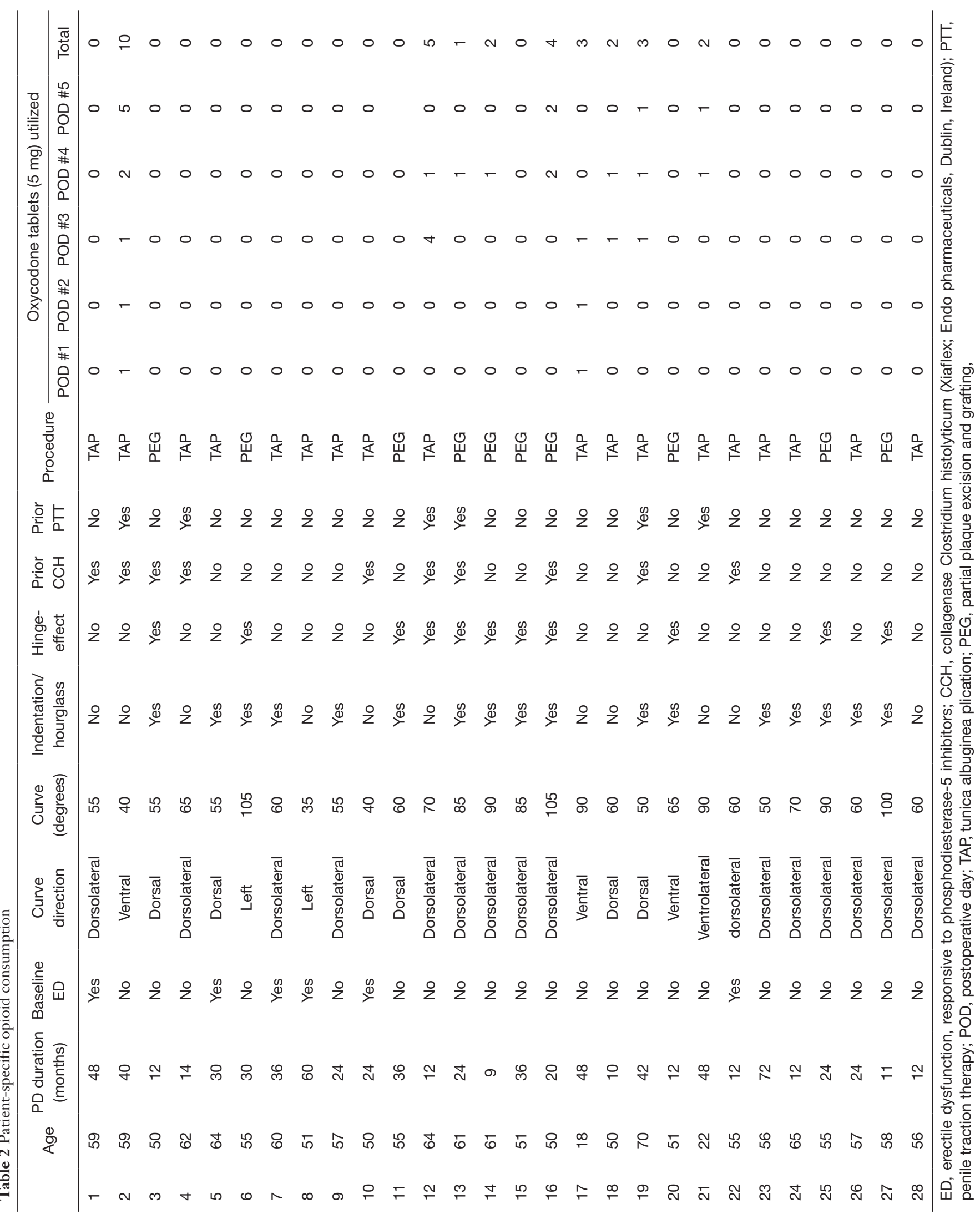


represents a desirable alternative with a longer duration of action.

Peri-incisional blocks with LB demonstrate considerable success in controlling post-operative pain for various surgical procedures, although data specific to urology is limited $(16,17,35,36)$. The safety profile of this medication is excellent $(37,38)$. Taniguchi et al. showed that intracavernosal LB injection at the time of penile prosthesis surgery did not result in any significant hemodynamic effects intraoperatively (39). Data supporting efficacy in urologic surgery was first reported by Cotta et al. in 2016 (35). They evaluated LB in a cohort of men who underwent penile prosthesis placement. The authors found that patients who received local anesthesia with LB administered in the corpora cavernosa, within the scrotum, and at the level of the inguinal ring $(\mathrm{n}=13)$ used significantly fewer opioids postoperatively when compared to men receiving standard bupivacaine blocks or no local anesthesia $(n=24)$. LB has also been described for use with in-office urologic procedures including hydrocelectomy, testicular sperm extraction, and vasectomy reversal with satisfactory peri- and postprocedural anesthesia reported $(36,40)$. Unfortunately, these descriptions did not quantify postoperative pain control including opioid use.

PD surgery offers an ideal opportunity for longacting local anesthetic blocks. Postoperative pain tends to be most prominent early-on within the first few days, but abates quickly. Due to the anatomical basis of penile innervation, infiltration with local anesthetic is technically easy and excellent pain control can be achieved easily and reliably with a penile ring block $(23,24)$. In fact, it has been suggested that more invasive penile surgeries including partial penectomy are possible with an adequate block (40). Our early findings suggest that preventing pain in the early window may allow us to avoid stronger oral analgesics including opioids in the majority of patients. Additional tangible benefits are also possible. For example, patients taking opioids are instructed to avoid driving, operating machinery, and work-related activities due to the centraleffects of these medications. If opioid use, and pain in general, was minimized, LB may actually allow patients to return to work immediately after surgery - possibly within the first day or two.

One important finding here was that nearly $90 \%$ of prescribed opioids were unnecessary with our protocol, despite the relatively small quantity of oxycodone that was prescribed (75 oral morphine equivalents). In comparison, previous reports in the urologic literature have found that roughly $60 \%$ of opioids go unused after other interventions $(27,28)$. The extent of over-prescribing in our small cohort was even greater if we consider the fact that three of the nine patients (33\%) who consumed opioids reported using the medication pre-emptively to ward off future pain, rather than as intended for breakthrough pain. All three patients felt that taking the oxycodone was unnecessary in retrospect. These findings have important societal implications. Prescription opioids may be diverted for misuse or abuse by persons other than the patient to whom they were prescribed. Based on these results, we suggest that prescription opioids appear unnecessary in most instances if a long-acting LB penile block is used during PD surgery, and prescribing opioids should be reserved for special circumstances.

Interestingly, the only factor that was associated with a greater likelihood of using opioids postoperatively was use of preoperative penile traction. The reasons for this finding are unclear, and may simply be due to sampling error given our small cohort size. Alternatively, we hypothesize that patients who use traction preoperatively may have an altered threshold for discomfort thereby impacting their perceived need for stronger oral analgesics after surgery.

Our study is not without notable limitations including the small sample size which lacks statistical power to identify potential factors associated with opioid use. There is no control group with which to compare postoperative opioid use after administration of shorter-acting local anesthetics. Therefore, we do not truly know if LB limits opioid use relative to alternative shorter acting local anesthetics. Future comparative studies are needed. We also relied on patient recall regarding the quantity of opioids consumed which introduces bias, and we lack data on postoperative pain scores beyond the initial immediate postoperative assessments. Finally, in the current era of increasing medical expenditures, LB remains a more expensive option at $\$ 180$ for $133 \mathrm{mg}(10 \mathrm{~mL})$ and $\$ 334$ for $255 \mathrm{mg}(20 \mathrm{~mL})$ compared to short-acting bupivacaine ( $\$ 3$ per dose), and this medication is not readily available at all institutions $(41,42)$. Despite these limitations, our results demonstrate the utility of long-acting LB to enhance pain control and limit opioid use following plication and grafting surgery for PD. 


\section{Conclusions}

This represents the first study to assess postoperative outcomes with long-acting LB in patients undergoing surgical correction for PD. Here, we demonstrate effective minimization of post-operative opioid use. Further studies, including a randomized trial comparing outcomes between short and long-acting local anesthetics, are necessary to determine whether the additional monetary cost of this agent is mitigated by the purported benefits with respect to pain control.

\section{Acknowledgments}

Funding: None.

\section{Footnote}

Reporting Checklist: The authors have completed the STROBE reporting checklist. Available at http://dx.doi. org/10.21037/tau-20-871

Data Sharing Statement: Available at http://dx.doi. org/10.21037/tau-20-871

Conflicts of Interest: All authors have completed the ICMJE uniform disclosure form (available at http://dx.doi. org/10.21037/tau-20-871). Dr. Kohler serves as an unpaid Associate Editor-in-Chief of Translational Andrology and Urology from January 2020 to December 2021. The other authors have no conflicts of interest to declare.

Ethical Statement: The authors are accountable for all aspects of the work in ensuring that questions related to the accuracy or integrity of any part of the work are appropriately investigated and resolved. The study was conducted in accordance with the Declaration of Helsinki (as revised in 2013). The study was approved by institutional review board of Mayo Clinic (No. 20-003836) and informed consent was taken from all individual participants.

Open Access Statement: This is an Open Access article distributed in accordance with the Creative Commons Attribution-NonCommercial-NoDerivs 4.0 International License (CC BY-NC-ND 4.0), which permits the noncommercial replication and distribution of the article with the strict proviso that no changes or edits are made and the original work is properly cited (including links to both the formal publication through the relevant DOI and the license). See: https://creativecommons.org/licenses/by-nc-nd/4.0/.

\section{References}

1. 2019 Annual Surveillance Report of Drug-Related Risks and Outcomes - United States Surveillance Special Report. Centers for Disease Control and Prevention, U.S. Department of Health and Human Services.

2. Siglin J, Sorkin JD, Namiranian K. Incidence of Postoperative Opioid Overdose and New Diagnosis of Opioid Use Disorder Among US Veterans. Am J Addict 2020;29:295-304.

3. Brummett CM, Waljee JF, Goesling J, et al. New Persistent Opioid Use After Minor and Major Surgical Procedures in US Adults. JAMA Surg 2017;152:e170504.

4. Maxwell JC. The prescription drug epidemic in the United States: a perfect storm. Drug Alcohol Rev 2011;30:264-70.

5. Shkolyar E, Shih IF, Li Y, et al. Robot-assisted radical prostatectomy associated with decreased persistent postoperative opioid use. J Endourol 2020;34:475-81.

6. Tam CA, Dauw CA, Ghani KR, et al. New persistent opioid use after outpatient ureteroscopy for upper tract stone treatment. Urology 2019;134:103-8.

7. Berger I, Strother M, Talwar R, et al. National variation in opioid prescription fills and long-term use in opioid naïve patients after urological surgery. J Urol 2019;202:1036-43.

8. Welk B, McClure JA, Clarke C, et al. An opioid prescription for men undergoing minor urologic surgery is associated with an increased risk of new persistent opioid use. Eur Urol 2020;77:68-75.

9. Shah AS, Blackwell RH, Kuo PC, et al. Rates and Risk Factors for Opioid Dependence and Overdose after Urological Surgery. J Urol 2017;198:1130-36.

10. Xu W, Daneshmand S, Bazargani ST, et al. Postoperative Pain Management after Radical Cystectomy: Comparing Traditional versus Enhanced Recovery Protocol Pathway. J Urol 2015;194:1209-13.

11. Meyer LA, Lasala J, Iniesta MD, et al. Effect of an Enhanced Recovery After Surgery Program on Opioid Use and Patient-Reported Outcomes. Obstet Gynecol 2018;132:281-90.

12. Saidian A, Nix JW. Enhanced Recovery After Surgery: Urology. Surg Clin North Am 2018;98:1265-74. 
13. Becker DE, Reed KL. Local anesthetics: a review of pharmacological considerations. Anesth Prog 2012;59:90-101.

14. Hansen TG. Ropivacaine: a pharmacological review. Expert Rev Neurother 2004;4:781-91.

15. FDA Label Approved on 10/28/2011 (PDF) for EXPAREL. US Silver Spring, MD: US Food and Drug Administration. Available online: http://www.accessdata. fda.gov/drugsatfda_docs/label/2011/022496s000lbl.pdf. Accessed 4/3/2020

16. Golf M, Daniels SE, Onel E. A phase 3, randomized, placebo-controlled trial of DepoFoam(R) bupivacaine (extended-release bupivacaine local analgesic) in bunionectomy. Adv Ther 2011;28:776-88.

17. Gorfine SR, Onel E, Patou G, et al. Bupivacaine extendedrelease liposome injection for prolonged postsurgical analgesia in patients undergoing hemorrhoidectomy: a multicenter, randomized, double-blind, placebo-controlled trial. Dis Colon Rectum 2011;54:1552-9.

18. Ellis JL, Higgins AM, Simhan J. Pain management strategies in penile implantation. Asian J Androl 2020;22:34-8.

19. Greenfield JM, Lucas S, Levine LA. Factors affecting the loss of length associated with tunica albuginea plication for correction of penile curvature. J Urol 2006;175:238-41.

20. Hatzichristodoulou G, Tsambarlis P, Kubler H, et al. Peyronie's graft surgery-tips and tricks from the masters in andrologic surgery. Transl Androl Urol 2017;6:645-56.

21. Levine LA, Lenting EL. A surgical algorithm for the treatment of Peyronie's disease. J Urol 1997;158:2149-52.

22. Nehra A, Alterowitz R, Culkin DJ, et al. Peyronie's Disease: AUA Guideline. J Urol 2015;194:745-53.

23. Broadman LM, Hannallah RS, Belman AB, et al. Postcircumcision analgesia- a prospective evaluation of subcutaneous ring block of the penis. Anesthesiology 1987;67:399-402.

24. Szmuk P, Ezri T, Ben Hur H, et al. Regional anaesthesia for circumcision in adults: a comparative study. Can J Anaesth 1994;41:1181-4.

25. Baskin LS, Duckett JW. Dorsal tunica albuginea plication for hypospadias curvature. J Urol 1994;151:1668-71.

26. Myles PS, Myles DB, Galagher W, et al. Measuring acute postoperative pain using the visual analog scale: the minimal clinically important difference and patient acceptable symptom state. Br J Anaesth 2017;118:424-9.

27. Theisen KM, Myrga JM, Hale N, et al. Excessive opioid prescribing after major urologic procedures. Urology 2019;123:101-7.

28. Bates C, Laciak R, Southwick A, et al. Overprescription of postoperative narcotics: a look at postoperative pain delivery, consumption and disposal in urological practice. J Urol 2011;185:551-5.

29. Lucas J, Gross M, Yafi F, et al. A Multi-institutional Assessment of Multimodal Analgesia in Penile Implant Recipients Demonstrates Dramatic Reduction in Pain Scores and Narcotic Usage. J Sex Med 2020;17:518-25.

30. Ohnuma T, Raghunathan K, Moore S, et al. Dosedependent association of gabapentinoids with pulmonary complications after total hip and knee arthroplasties. J Bone Joint Surg Am 2020;102:221-9.

31. Ashburn MA, Fleisher LA. Do gabapentinoids cause more harm than good? Ann Surg 2019;270:e68.

32. Thornblade LW, Seo YD, Kwan T, et al. Enhanced Recovery via Peripheral Nerve Block for Open Hepatectomy. J Gastrointest Surg 2018; 22:981-8.

33. Mortensen K, Nilsson M, Slim K, et al. Consensus guidelines for enhanced recovery after gastrectomy: enhanced recovery after surgery (ERAS) society recommendations. Br J Surg 2014;101:1209-29.

34. Wick EC, Grant MC, Wu CL. Postoperative multimodal analgesia pain management with nonopioid analgesics and techniques: a review. JAMA Surg 2017;152:691-7.

35. Cotta BH, Welliver C, Brahmamdam A, et al. Longacting liposomal bupivacaine decreases inpatient narcotic requirements in men undergoing penile prosthesis implantation. Turk J Urol 2016;42:230-4.

36. Ziegelmann M, Dodge N, Alom M, et al. Office-based, Minimal-Incision Modified Fenestration Technique for Symptomatic Hydroceles Under Local Anesthesia. Urology 2020;135:159-64.

37. Vyas KS, Rajendran S, Morrison SD, et al. Systematic Review of Liposomal Bupivacaine (Exparel) for Postoperative Analgesia. Plast Reconstr Surg 2016;138:748e-56e.

38. Malik O, Kaye AD, Kaye A, et al. Emerging roles of liposomal bupivacaine in anesthesia practice. J Anaesthesiol Clin Pharmacol 2017;33:151-6.

39. Taniguchi H, Mulhall JP. Intraoperative Intracavernosal Liposomal Bupivacaine (Exparel) Injection Does Not Affect Systemic Hemodynamics. J Sex Med 2020;17:526-30.

40. Alom M, Ziegelmann M, Savage J, et al. Office-based 
andrology and male infertility procedures- a cost-effective alternative. Transl Androl Urol 2017;6:761-72.

41. Young WY. New study supports exparel cost effectiveness. This Week Orthopedics. Published January 24th, 2018. Accessed April 9th, 2020. Available online: https:// ryortho.com/breaking/new-study-supports-exparel-cost-

Cite this article as: Britton CJ, Findlay BL, Parikh N, Kohler T, Helo S, Ziegelmann MJ. Long-acting liposomal bupivacaine and postoperative opioid use after Peyronie's disease surgery: a pilot study. Transl Androl Urol 2021;10(1):174183. doi: $10.21037 /$ tau-20-871 effectiveness/

42. Clinical and economic value outcomes with Exparel. Exparel. Pacira BioSciences, Inc. 2020. Accessed April 9th, 2020. Available online: https://www.exparel.com/hcp/ value/colorectal-surgery 